\title{
Fundamentos del Diseño Universal para el Aprendizaje Desde la Perspectiva InTERnaCionaL ${ }^{1}$ \\ Fundamentals of UNIVERSAL DESIGN FOR LEARNING FROM AN INTERNATIONAL \\ PERSPECTIVE
}

\author{
Mario CORTÉS DÍAZ² \\ Camino FERREIRA VILLA ${ }^{3}$ \\ Ana Rosa ARIAS GAGO ${ }^{4}$
}

\begin{abstract}
RESUMEN: El presente estudio se enmarca en el ámbito educativo, concretamente, en el área de la educación inclusiva, profundizando en el Diseńo Universal para el Aprendizaje (DUA). El DUA se concibe como un enfoque teórico y práctico que actúa como una herramienta eficaz para alcanzar una educación de calidad y equidad dentro del movimiento de la inclusión educativa. El objetivo de este estudio es analizar la presencia de los fundamentos del DUA (derecho de la educación; diversidad; Diseño Universal; currículo flexible; igualdad de oportunidades; neurociencia y tecnologías de la información y la comunicación) en los documentos publicados por los organismos internacionales con competencia en materia educativa y de repercusión mundial. La metodología de la investigación es de corte cualitativo utilizando el análisis documental. Las categorías de análisis corresponden a los siete fundamentos del DUA. Las fuentes documentales para el estudio han sido fundamentalmente las páginas web de los organismos internacionales y la base de datos UNESDOC, obteniéndose una muestra definitiva de once documentos pertenecientes a la literatura gris. Tras la revisión documental, los resultados han evidenciado que no todos los documentos objeto de estudio contemplan en su contenido las categorías de análisis. En suma, se observan carencias en cuanto a la presencia de los siete fundamentos del DUA en los documentos publicados analizados, por lo que se requiere de un nuevo tratamiento a nivel internacional del DUA con el fin de promover una educación de calidad e inclusiva, que atiende y respeta a las diferencias individuales dentro del proceso de enseńanza-aprendizaje.
\end{abstract}

PALABRAS CLAVE: Educación. Inclusión. Diversidad. Organización internacional.

\begin{abstract}
The present study was carried out in the frame of the educational field, specifically in the area ofinclusive education, going deeper into the Universal Design for Learning (UDL). UDL is conceived as a theoretical and practical approach that acts as an effective tool to achieve quality education and equity within the educational inclusion movement. The objective of this study is to analyze the presence of the fundamentals of UDL (education law; diversity; Universal Design; flexible curriculum; equal opportunities; neuroscience and information and communication technologies) in documents published by international organizations with competence in education and global impact. The research methodology is of a qualitative nature and uses documentary analysis. The categories of analysis correspond to the seven fundamentals of UDL. The documentary sources of the study were mainly the web pages of international organizations and the UNESDOC database, obtaining a definitive sample of eleven documents belonging to the gray literature. After the document review, the results showed that not all documents under study include the categories of analysis in their content. In summary, there are gaps in the presence of the seven fundamentals of UDL in the published documents analyzed, which is why a new international treatment of the UDL is needed to promote an inclusive and quality education that meets and respects individual differences within the teaching-learning process.
\end{abstract}

KEYWORDS: Education. Inclusion. Diversity. International organization.

\footnotetext{
${ }^{1}$ https://doi.org/10.1590/1980-54702021v27e0065

${ }^{2}$ Universidad de León. Facultad de Educación. León/España. E-mail: mcortd00@estudiantes.unileon.es. ORCID: https://orcid. org/0000-0003-1280-0913

${ }^{3}$ Universidad de León. Departamento de Psicología, Sociología y Filosofía. León/Espańa. E-mail: camino.ferreira@unileon.es. ORCID: https://orcid.org/0000-0001-8131-0825

${ }^{4}$ Universidad de León. Departamento de Didáctica General, Específicas y Teoría de la Educación. León/Espańa. E-mail: ana. arias@unileon.es. ORCID: https://orcid.org/0000-0002-5889-3222
} 


\section{INTRODUCCIÓN}

El Diseño Universal para el Aprendizaje (DUA) se concibe como un enfoque educativo de intervención que se enmarca dentro del movimiento de la educación inclusiva. Históricamente, la inclusión tiene sus orígenes en el movimiento de la educación regular que apareció en Estados Unidos con el objetivo de incluir a los niños con algún tipo de discapacidad en las escuelas ordinarias. Según Torres (2010) todos los alumnos, sin excepciones, deben estar escolarizados en las aulas regulares para que puedan recibir una educación eficaz. La aportación de este autor deja entrever que una de las vías de atención educativa, años atrás, era la integración, pero la educación inclusiva, ofrece una visión más amplia (Parra, 2011). La educación inclusiva se encuentra en constante evolución concentrándose la mayor proliferación de artículos entre los años 2012 y 2013 (Suárez \& López, 2018), siendo actualmente uno de los compromisos de las políticas de la Unión Europea (Alba, 2019).

Teniendo como referencia la educación inclusiva, asentamos las bases del DUA, el cual surge en el Centro de Tecnología Especial Aplicada (en inglés, CAST) en el año 1984. El planteamiento DUA presenta una serie de fundamentos que hacen que este enfoque se sitúe como una herramienta eficaz para alcanzar una educación inclusiva y de calidad.

\subsection{DUA y Derecho a la EdUCACión}

Desde el marco DUA todos los alumnos tienen derecho a la educación, y ésta debe darse bajo unas condiciones en las cuales todos tengan las mismas opciones para aprender independientemente de si presentan discapacidad o no. El reconocimiento universal de la educación como derecho se remonta al año 1948 cuando se proclama la Declaración Universal de los Derechos Humanos (Organización de las Naciones Unidas [ONU], 1948). La ratificación de este derecho será de nuevo expuesto por la Convención de los Derechos del Niño (Organización de las Naciones Unidas [ONU], 1989).

Teniendo como referencia ambos documentos, la filosofía DUA plantea un diseño curricular flexible en tanto al método, materiales y evaluación, que se fundamenta en la consideración de la educación como un derecho. Este planteamiento se encuentra en consonancia con las ideas de la educación inclusiva porque avala el derecho a la educación y promueve la igualdad de las condiciones de vida (Mariussi et al., 2016). Además, el movimiento de la inclusión permite avanzar hacia la educación para todos y garantizar el derecho a una educación (Blanco, 2006).

\subsection{DUA y DiVERSIDAD}

Según Alba (2019), el DUA contempla un marco teórico y práctico en el campo educativo para atender a la diversidad desde un enfoque inclusivo que permita la aceptación y reconocimiento de las necesidades y diferencias individuales de los alumnos. La consideración de la diversidad en términos positivos conlleva que uno de los desafíos actuales del sistema educativo sea dar respuesta a una sociedad cada vez más cambiante y diversa. En esta línea de trabajo centra su atención el DUA, ya que constituye un enfoque educativo basado en la valoración de la diversidad como elemento enriquecedor del proceso de enseñanza-aprendizaje, contribuyendo al desarrollo humano (Parra, 2011). Atendiendo a esta consideración, cada alu- 
mno presenta unas necesidades educativas y, la manera que tienen de enfrentarse al aprendizaje, es muy diferente entre ellos (Blanco, 2006).

\subsection{DUA, DU y Currículo}

El marco DUA y el Diseño Universal se encuentran en consonancia porque desde el DUA se incorpora al currículo los principios del Diseño Universal (DU). El DU es un movimiento que procede del ámbito de la arquitectura y cuyo pionero fue el arquitecto Mace (1985) en colaboración con la Universidad de Carolina del Norte (Burgstahler, 2008). La finalidad del DU es crear entornos que pudieran ser utilizados por todas las personas y no sólo por aquellas que presentaran algún tipo de discapacidad, incluyéndose los entornos educativos. Dentro de esta área, el DU actúa como el medio más adecuado para garantizar el acceso a la educación para todos mediante un modelo pedagógico que tiene como eje la inclusión y equidad para la creación de materiales accesibles (Schelly et al., 2011).

La presencia de los siete principios del DU (uso equiparable; uso flexible; simple e intuitivo; información perceptible; tolerancia al error; que exija poco esfuerzo físico y tamaño y espacio apropiados para el acceso) dados por el Centro para el Diseño Universal (Center for Universal Design [CUD], 1997) permiten desde el planteamiento del DUA la creación de entornos de aprendizaje en los cuales prima un currículo flexible, en tanto objetivos, contenidos, materiales y evaluación, que permite derribar los obstáculos que ofrecen los currículos inflexibles.

\subsection{DUA E IGUALDAD DE OPORTUNIDADES}

En el contexto internacional, el DUA ha supuesto un avance en derechos sobre igualdad de oportunidades (Ruiz-Bel et al., 2012). Este fundamento en el marco DUA se contextualiza a través del diseño de currículos flexibles que son la clave para dar respuesta a la variabilidad de los alumnos y garantizar la igualdad de oportunidades. Esto es un ejemplo más de que el DUA se encuentra en consonancia con la educación inclusiva. El planteamiento DUA proporciona oportunidades de aprendizaje a todos los estudiantes (Alba, 2019). Así pues, la creación de entornos de aprendizaje en los cuales no tengan cabida las barreras ni la exclusión, se sitúa al DUA como un enfoque en el que prima la igualdad de oportunidades como fundamento de actuación porque, sólo así, se podrá garantizar una educación de calidad en la que se tenga en cuenta el desarrollo del alumno, así como su identidad, cultura o idioma.

\subsection{DUA Y NEUROCIENCIA}

El planteamiento DUA avala sus investigaciones en la neurociencia, y, por tanto, será esta ciencia un punto de referencia y fundamento en el marco DUA. Tomando como referencia los avances neurocientíficos, los investigadores Rose y Meyer (2006) determinaron la existencia tres tipos de subredes cerebrales: (1) Red de conocimiento, (2) Red estratégica y (3) Red afectiva. Estas redes intervienen en el proceso de aprendizaje y están especializadas en tareas específicas del procesamiento de la información o ejecución. La presencia de dichas redes cerebrales mantiene una estrecha relación con los tres principios del marco DUA. Las investigaciones del Centro de Tecnología Especial Aplicada ([CAST], 2011) determinan tres principios: (1) Proporcionar múltiples formas de representación de la información y los contenidos, 
(2) Proporcionar múltiples formas de expresión del aprendizaje y (3) Proporcionar múltiples formas de implicación.

La importancia de la neurociencia en el mundo educativo radica en su labor como herramienta para conocer y entender cómo funciona el aprendizaje a través del estudio del cerebro. Concretamente, la neurociencia tiene como objeto de estudio el sistema nervioso, y cómo la actividad cerebral se relaciona con el aprendizaje y la conducta (Salas, 2003).

\subsection{DUA y TeCnologías de la Información y la CoMUnicación (TIC)}

Una de las herramientas que utiliza el DUA para eliminar las barreras que ofrecen los currículos inflexibles son las Tecnologías de la Información y la Comunicación (TIC). En esta línea, se aconseja que los medios digitales se adapten a los capacidades, motivaciones y habilidades y estilo de aprendizaje. De ese modo, se consigue que se minimicen los obstáculos que pueden presentan los medios tradicionales ya que estos presentan un único modelo. Además, Rose y Meyer (2002) consideran que las TIC son un pilar fundamental dentro del planteamiento DUA para poder garantizar una educación de calidad e inclusiva. En consonancia con el DUA, la educación inclusiva requiere de la utilización de las TIC (Zulian \& Freitas, 2001), facilitando la presencia de contextos de aprendizaje en los que se tenga en cuenta la diversidad del alumnado (Fernández-Batenero \& Arrifano, 2019).

Tras lo expuesto, el DUA se apoya en una serie de fundamentos que dan sentido a la finalidad de su planteamiento. Estos fundamentos se concretan en la consideración de la educación como un derecho, la diversidad, la construcción de un diseño educativo basado en los principios del DU, un currículo flexible, la igualdad de oportunidades, la fundamentación de la neurociencia y la utilización de las TIC. Según Hartmann (2011), con el DUA estamos ante una nueva manera de pensar la educación, pero ¿se han reflejado los fundamentos del DUA en los documentos publicados por las organizaciones internacionales en materia educativa de los últimos ańos? Para responder a esta pregunta, nuestro objetivo se centra en analizar cuál es y cómo se refleja la presencia de los fundamentos del marco DUA en el contexto internacional dentro del área de la educación inclusiva.

\section{MéTodo}

La metodología de la investigación es de corte cualitativa mediante el uso del análisis documental. Según Payne y Payne (2004), este método permite analizar documentos oficiales y primarios acerca del fenómeno que se quiere estudiar. Para este estudio, se determinará la presencia de los fundamentos del DUA en los diferentes documentos publicados por los organismos internacionales con competencia en materia educativa en el marco de la educación inclusiva. El desarrollo metodológico del presente artículo consta de dos partes. La primera de ellas ha consistido en identificar y seleccionar los organismos internacionales con atribuciones en materia educativa y de relevancia en el ámbito de la educación inclusiva. Para ello, se ha tomado en consideración dos documentos: (1) Principios fundamentales para la promoción de la calidad de la educación inclusiva. Recomendaciones a responsables politicos, elaborado por Watkins (2009) para la Agencia Europea para el Desarrollo de la Educación Especial; y (2) el documento Mejor Educación para todos, un informe mundial elaborado por el Instituto Universitario de 
Integración en la Comunidad (2009). Así mismo, se ha tenido en cuenta la fuente "La inclusión educativa como proceso en contextos socioeducativos" de la Universidad Nacional de Educación a Distancia (2018) para su contrastación y actualización.

Tras la revisión de los trabajos anteriormente citados se han seleccionado tres organismos: (1) Organización de las Naciones Unidas para la Educación, la Ciencia y la Cultura (UNESCO), (2) Organización de Naciones Unidas (ONU) y (3) Agencia Europea para el Desarrollo de la Educación del Alumnado con Necesidades Educativas Especiales. La elección de estas organizaciones viene motivada por su trascendencia a nivel internacional en materia educativa y su especial dedicación al ámbito de la educación inclusiva.

Una vez identificados los organismos internacionales, la segunda parte del método ha consistido en seleccionar los tipos de documentos divulgados por dichos organismos. Para este estudio se ha decidido seleccionar literatura gris referida a declaraciones, convenciones, conferencias y foros publicados en español y cuya temática es la educación inclusiva. Se excluyen de esta selección las actas de congresos y jornadas, así como tesis doctorales, ya que su naturaleza no se contempla como objeto de este estudio.

La búsqueda de los documentos se ha realizado a través de dos fuentes principales: UNESDOC y las páginas web de los organismos seleccionados; haciendo uso de los descriptores "conferencia de educación inclusiva"; "foro de educación inclusiva" y "declaración de educación inclusiva”. Así mismo, se han excluido los documentos duplicados y los que no tuvieran relación con la naturaleza del objeto de estudio.

El proceso de selección y análisis de los documentos identificados es el siguiente: número de registros identificados $(\mathrm{n}=48)$; número de registros tras eliminar citas duplicadas $(\mathrm{n}=29)$; número de registros cribados $(\mathrm{n}=29)$; número de registros excluidos $(\mathrm{n}=16)$; número de documentos evaluados para su elegibilidad $(n=13)$; número de documentos excluidos por razones de contenido que no se relacionaba con el tema de estudio o no encajaba dentro del análisis basado en los fundamentos del $\operatorname{DUA}(n=2)$; número de documentos incluidos en la síntesis cualitativa $(n=11)$. En total, la muestra de fuentes documentales analizadas se compone de once documentos, marcados con un "*” en las referencias bibliográficas.

\section{Resultados}

En este apartado presentamos los datos de las siete categorías de análisis establecidas en función de los fundamentos del DUA: educación como un derecho; diversidad; DU; currículo flexible; igualdad de oportunidades; neurociencia y TIC.

En la Tabla 1 podemos observar tres grandes columnas. La primera de ellas hace referencia al número de documento, la segunda refleja el año de publicación y, la tercera describe el organismo internacional que lo ha publicado. Las sucesivas columnas se identifican con las categorías de análisis (fundamentos del DUA). En estas casillas se consigna con el símbolo ".” si el documento objeto de revisión presenta la categoría de análisis. 


\section{Tabla 1}

Relación de los Fundamentos del DUA y los documentos analizados de los Organismos Internacionales
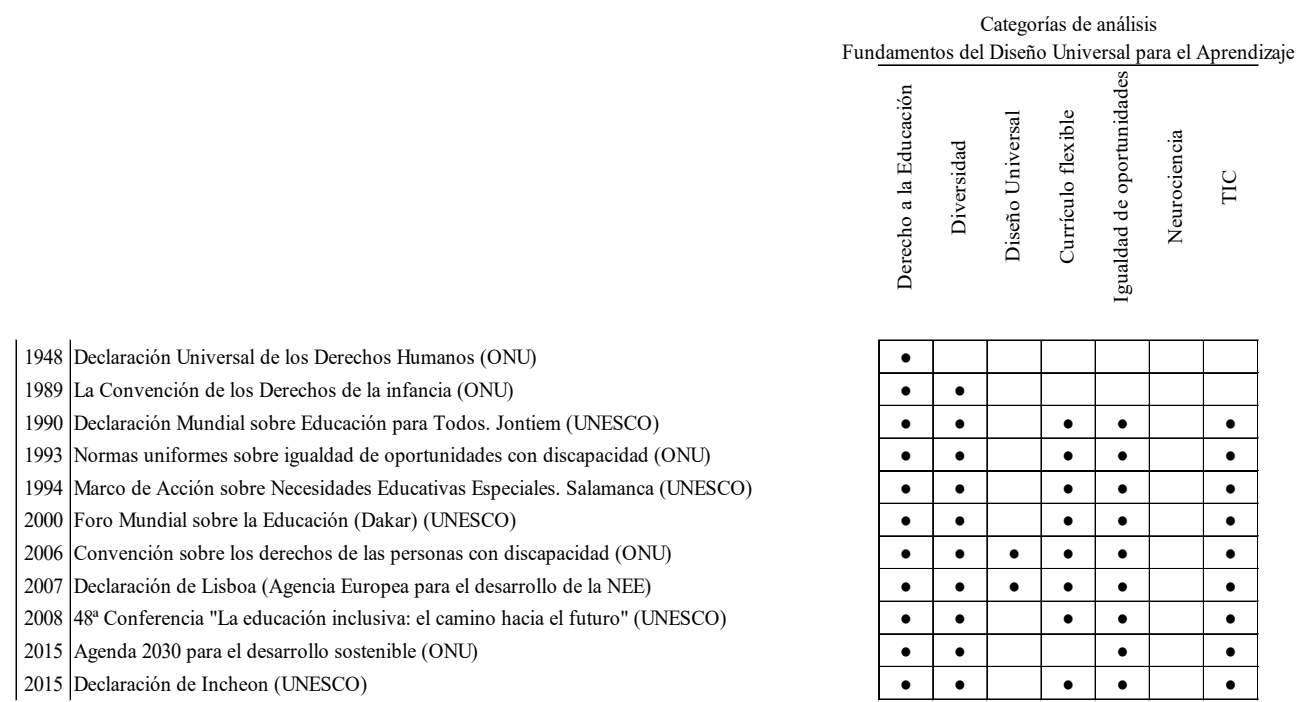

Los resultados se presentan de acuerdo a las categorías de análisis establecidas, las cuales responden a los fundamentos del DUA. A lo largo del análisis se nombra el documento y entre paréntesis su correspondencia numérica, tal y como aparece en la Tabla 1.

\subsection{Derecho a la Educación}

En relación a la categoría derecho a la educación, ésta aparece por primera vez en la Declaración Universal de los Derechos Humanos (1) (Organización de las Naciones Unidas [ONU], 1948). Este documento supone un hito fundamental en la historia de la humanidad por la trascendencia legal que ha tenido en muchos países. La citada Declaración a través de su artículo 26.1 enuncia que "toda persona tiene derecho a la educación" (ONU, 1948); siendo la referencia para el resto de documentos objeto de este estudio. Como podemos observar en la Tabla 1, la categoría "Derecho a la Educación" es la de mayor frecuencia en términos de presencia en los documentos vinculados con la educación inclusiva. Años más tarde, la Convención sobre los Derechos del Niño (2) (ONU, 1989), en su artículo 29 expresa "los Estados Partes reconocen el derecho del niño a la educación y, a fin de que se pueda ejercer progresivamente y en condiciones de igualdad de oportunidades ese derecho" (ONU, 1989). Otro ejemplo es el Marco de Acción sobre Necesidades Educativas Especiales (5) (Naciones Unidas para la Educación, la Ciencia y la Cultura [UNESCO], 1994) en el cual se expone en relación a esta categoría que "todos los niños de ambos sexos tienen un derecho fundamental a la educación" (UNESCO, 1994. p. 8).

Aunque el DUA no había hecho su aparición en el campo de la educación en el momento en el que se dio a conocer la Declaración Universal de los Derechos Humanos (1) (ONU, 1948), contempla entre sus fundamentos la consideración de la educación como un 
derecho, entendida ésta desde la concepción de una educación para todos independientemente de sus condicionantes sociales, económicos, culturales, de raza, sexo y/o religión. Años más tarde, en la Convención sobre los derechos de las personas con discapacidad (7) indica que "los Estados Partes reconocen el derecho de las personas con discapacidad a la educación” (ONU, 2006, art. 24).

El resto de documentos no sólo hace referencia a lo expuesto en el artículo 26.1 de la Declaración Universal de los Derechos Humanos (1) (ONU, 1948), sino que de ellos se desprende que la educación es el medio más adecuado para contribuir al desarrollo al desarrollo personal y profesional del alumno. Tras el paso de los años, esta conceptualización se ha visto enriquecida, ya que en la Declaración Mundial sobre Educación para Todos de Jontiem (3) se expone que "la educación es la transmisión y el enriquecimiento de los valores culturales y morales comunes" (UNESCO, 1990, art. 1). A lo largo de los diez artículos que conforman la Conferencia de Jontiem se hacen alusiones directas a la educación como un derecho y a la necesidad de satisfacer las necesidades básicas de aprendizaje. Quince años más tarde, la Declaración de Incheon (11) (UNESCO, 2015) atribuye a la educación un valor humanístico que comprende todos los ámbitos de la persona. Así pues, estamos ante una categoría fundamental dentro de los documentos objeto de estudio y es tomada en consideración por el marco DUA.

\subsection{Diversidad}

En relación a la categoría diversidad, el punto de partida lo concede la Convención de los Derechos del Niño (2) la cual expresa en su artículo 23 que "los Estados Partes reconocen que el niño mental o físicamente impedido deberá disfrutar de una vida plena y decente en condiciones que aseguren su dignidad" (ONU, 1989). Se da el primer paso en términos de reconocimiento universal a la consideración de la diversidad y el respeto a las diferencias individuales, con especial atención a la población con discapacidad. Un ejemplo de ello lo encontramos en la Declaración Mundial sobre Educación para Todos celebrada en Jontiem (3) donde se enuncia que "las necesidades básicas de aprendizaje de las personas impedidas precisan especial atención” (UNESCO, 1990, art. 3.5). Con ello, se creó la idea de educación para todos centrada en el aprendizaje en su sentido más amplio, aquel que tiene lugar durante toda la vida, y se produce un movimiento hacia la educación inclusiva.

Cuatro años más tarde tuvo lugar la Conferencia Mundial sobre Necesidades Educativas Especiales: Acceso y Calidad celebrada en Salamanca (5) (UNESCO, 1994). En dicho espacio se establecieron hitos muy significativos para la educación y la atención a alumnos con necesidades educativas especiales. El principio rector de su Marco de Acción es que los centros educativos deben escolarizar a todos los niños independientemente de sus condiciones. Los participantes se reafirmaron en la educación para todos, recordando lo que ya en 1990 se expuso en Jontiem. En Salamanca se adoptó como premisa de trabajo que es necesario impartir enseńanza a todos los alumnos con necesidades educativas especiales en el sistema común de educación. Esta consideración supondrá un punto de inflexión en el campo de las necesidades educativas especiales.

Con la llegada del nuevo milenio, el Foro Mundial de Educación (6) se reafirma en lo expuesto en la Declaración de Jontiem (3) (UNESCO, 1990) y centra su atención en la 
diversidad del siguiente modo: "una educación orientada a explotar los talentos y capacidades de cada persona y desarrollar la personalidad del educando, con objeto de que mejore su vida y transforme la sociedad" (UNESCO, 2000, p. 8). Las aportaciones de los documentos citados se encuentran en consonancia con el DUA porque este paradigma, dentro de sus fundamentos, tiene como pretensión ofrecer un diseño curricular adaptado a los alumnos que presenten discapacidad o no apoyándose en el uso de las TIC. Cabe señalar como observación que el CAST (1984), centro de referencia del DUA, comenzó sus estudios en el campo de la discapacidad desarrollando métodos para facilitar a los estudiantes con discapacidad el acceso al currículo general. Un ejemplo de ello es la transformación de los libros impresos en electrónicos. De lo expuesto anteriormente se deriva que los resultados encontrados en referencia a esta categoría es que la consideración de la diversidad es clave en los documentos objeto de estudio y de gran relevancia en el planteamiento del DUA.

\subsection{DisEÑo UNIVERSAL}

La categoría diseño universal es la que menor presencia tiene en los documentos objeto de estudio, apareciendo, tan solo en dos documentos, la Convención sobre los derechos de las personas con discapacidad (7) (ONU, 2006) y la Declaración de Lisboa (8) (Agencia Europea para el desarrollo de las Necesidades Educativas Especiales, 2007), los cuales centran su atención en el ámbito de la discapacidad. Resulta significativo la ausencia de esta categoría en el resto de documentos, ya que este enfoque, a pesar de haber nacido en el ámbito de la arquitectura, rápidamente se extendió al mundo educativo gracias al Centro para el Diseño Universal (CUD, 1997) formulando los siete principios del DU. Si bien es cierto, en el documento denominado Normas uniformes sobre igualdad de oportunidades con discapacidad (4) (ONU, 1993) se alude al DU, pero aplicado al entorno físico y no al campo educativo.

Años después, la Convención sobre los derechos de las personas con discapacidad (7) reconoció "la importancia de la accesibilidad al entorno físico, social, económico y cultural, a la salud y la educación y a la información y las comunicaciones" (ONU, 2006, p. 3). De hecho, esta Convención es la única que refleja una definición acerca del DU entendiendo por ello "el diseño de productos, entornos, programas y servicios que puedan utilizar todas las personas, en la mayor medida posible, sin necesidad de adaptación ni diseño especializado" (ONU, 2006, art. 2). Un ańo después, en la Declaración de Lisboa (8) (Agencia Europea para el Desarrollo de las Necesidades Educativas Especiales, 2007) se aborda la accesibilidad en términos de DU reclamando un mayor acceso en la educación y la sociedad.

\subsection{Currículo flexible}

Con respecto a la categoría currículo flexible se ha considerado para su análisis también el concepto equivalente de plan de estudios. Es en la Declaración Mundial sobre Educación para Todos (3) cuando se habla por primera vez de la necesidad de que los planes de estudio se ajusten a las necesidades de los alumnos y su diversidad, "de ahí que sea necesario determinar niveles aceptables de adquisición de conocimientos mediante el aprendizaje en los planes de educación y aplicar sistemas mejorados de evaluación de los resultados" (UNESCO, 1990, art. 
4). Esta consideración implica que los planes de estudio deben ser flexibles atendiendo a las diferencias individuales de los alumnos.

Cuatro años más tarde, la Declaración de Salamanca (5) subraya la necesidad de la puesta en marcha de planes de estudio adecuados a las necesidades de los alumnos y se centra en la necesidad de ofrecer a los alumnos opciones curriculares que se adapten a los niños con capacidades e intereses diferentes (UNESCO, 1994, p. 22). Por otro lado, las Normas uniformes sobre igualdad de oportunidades con discapacidad (4) indica que "la educación de las personas con discapacidad debe constituir parte integrante de la planificación nacional de la enseñanza, la elaboración de planes de estudio y la organización escolar” (ONU, 1993, art. 6).

Años más tarde tuvo lugar el Foro Mundial de Dakar (6), desde este Foro se enuncia la necesidad de que "los sistemas educativos deberán atender con flexibilidad a sus necesidades, facilitando contenidos adecuados de manera accesible y atractiva" (UNESCO, 2000). Esta consideración se encuentra en consonancia con el DUA, ya que a través de este enfoque se propone un diseño curricular flexible, en tanto a métodos, materiales y evaluación, para adaptarse a las necesidades y diferencias de los alumnos. Desde el enfoque DUA la consideración de un currículo flexible proporciona efectivas oportunidades de aprendizaje para todos a la vez que se potencia el aprendizaje significativo, lo que da lugar a aprendices expertos.

Seis años más tarde a la celebración del Foro Mundial (6), tuvo lugar la Convención sobre los derechos de las personas con discapacidad. A través de este documento se reconoce en el ámbito educativo, y en los planes de estudio, la necesidad de que "se hagan ajustes razonables en función de las necesidades individuales” (ONU, 2006, art. 24, p. 19). Un año después, en la Declaración de Lisboa (8) se aborda la necesidad de que las personas con discapacidad cuenten con una mayor flexibilidad, exponiendo que "en las clases y en los exámenes algunos necesitamos más tiempo" (Agencia Europea para el Desarrollo de las Necesidades Educativas Especiales, 2007, p. 2).

\subsection{IGUALDAD DE OPORTUNIDADES}

La Organización de las Naciones Unidas en el mes de diciembre de 1993 adoptaron las Normas Uniformes sobre la igualdad de oportunidades (4) (ONU, 1993) para personas con discapacidad como instrumento para la Unión Europea. Con la llegada del nuevo milenio, la Convención sobre los derechos de las personas con discapacidad (7) trabajó con el objetivo de garantizar la igualdad de oportunidades para todas las personas con discapacidad (ONU, 2006, art. 1, p. 4). Bajo esta línea se enmarca el DUA, ya que se presenta como un enfoque educativo e instrumento para la puesta en práctica de la educación inclusiva, que desarrolla sus planteamientos teniendo como premisa de actuación la igualdad de oportunidades y una educación para todos y, de cuya aplicación, se pueden beneficiar todos los miembros de la comunidad educativa.

Así mismo, el documento Agenda 2030 (10) (ONU, 2015) contempla entre uno de sus objetivos del desarrollo sostenible (ODS) el promover las mismas oportunidades de aprendizaje para todos. A través de este objetivo queda patente la importancia de la educación como medio para mejorar la vida de las personas y, como consecuencia, concibe la educación como la clave para eliminar las diferencias de género en la educación y garantizar el acceso en condiciones de igualdad de las personas vulnerables incluidas las personas con discapacidad, 
los pueblos indígenas y los niños en situaciones de vulnerabilidad a todos los niveles de la enseñanza y la formación profesional para poder alcanzar otros muchos ODS. Años atrás, la Declaración Mundial sobre Educación para Todos celebrada en Jontiem (3) estableció que "la prioridad más urgente es garantizar el acceso y mejorar la calidad de la educación para nińos y mujeres y en suprimir cuantos obstáculos se opongan a su participación activa” (UNESCO, 1990, art. 3.3). Cuatro años más tarde, la Declaración de Salamanca (5) recoge que "las escuelas integradoras representan un marco favorable para lograr la igualdad de oportunidades" (UNESCO, 1994, p. 11).

Posteriormente en el Foro Mundial de Dakar (6), se inicia un avance para romper con las desigualdades con el género femenino, en dicho Foro se expone "Mejorar la alfabetización de la mujer es otro factor esencial para promover la educación de las niñas” (UNESCO, 2000, p. 17). El Foro (UNESCO, 2000) centró la atención en la importancia de la educación de las nińas, en el aprendizaje de calidad y en la tarea de atender las necesidades de las personas que siguen estando marginadas en el ámbito de la educación. Estamos, pues, ante una de las primeras consideraciones sobre un movimiento basado en la igualdad de género que trasciende más allá del ámbito educativo. A partir de Dakar (2000) la preocupación por la igualdad de género será una constante. Seis años más tarde, la Convención sobre los derechos de las personas con discapacidad (7) lo explicita en su artículo 3 "la igualdad de oportunidades" (ONU, 2006, p. 5). Bajo esta categoría, el DUA puede ser una herramienta para contribuir al acceso igualitario a la educación, centrando su atención en el alumno y su manera de aprender y, no tanto, en la manera de enseńar del docente.

Hasta la aparición de la Convención de los Derechos de las personas con discapacidad (ONU, 2006) no han concurrido declaraciones internacionales legalmente que reconozcan, por ejemplo, a este colectivo el derecho a una educación sobre la base de la igualdad de oportunidades. Tres años más tarde, la Conferencia Internacional celebrada en Ginebra (9), y en referencia a la categoría igualdad de oportunidades expone que muchos países todavía presentan carencias en igualdad de oportunidades, a pesar de las altas tasas de escolarización de algunos de ellos (UNESCO, 2008, p. 6). Posteriormente, en la Declaración de Incheon (11) se expresa que "reconocemos la importancia de la igualdad de género para lograr el derecho a la educación para todos" (UNESCO, 2015, p. 2). En ese mismo año, la Agenda 203 (10) reconoce que "de aquí a 2030, asegurar el acceso igualitario de todos los hombres y las mujeres a una formación técnica, profesional y superior de calidad, incluida la enseñanza universitaria” (ONU, 2015, p. 19).

\subsection{Neurociencia}

La siguiente categoría que vamos a revisar pertenece a la neurociencia. Es muy significativa la ausencia de esta categoría en todos los documentos revisados, puesto que los avances en la neurociencia han servido al marco DUA para identificar tres redes cerebrales que intervienen en el procesamiento de la información y en los procesos de aprendizaje. Las aportaciones en esta línea de trabajo del marco DUA indican que el cerebro del ser humano es racional y emocional. Es por esto que el alumno aprende mejor aquello que nos aporta algo emocionalmente. Esta visión permite a la neurociencia adaptarse a cada alumno y atender a sus nece- 
sidades de aprendizaje. Es revelador que los documentos, especialmente los publicados en el nuevo milenio, han obviado la importancia que tiene hoy en día la neurociencia, centrándose en mayor medida en teorías cognitivas. Así pues, el DUA apuesta por la importancia que adquiere la plasticidad cerebral en el aprendizaje y los conocimientos en constante cambio, pero, también por la necesidad de flexibilización que contribuye al desarrollo del máximo potencial de aprendizaje del alumnado.

\subsection{TeCnologías de la Información y la CoMUNiCACión}

Por último, la importancia de las TIC en el ámbito educativo se indica a nivel internacional en el año 1990 con la Declaración Mundial sobre Educación para Todos que "los medios tradicionales, pueden movilizarse otros como las bibliotecas, la televisión y la radio, con el fin de utilizar sus posibilidades para satisfacer las necesidades de educación básica todos" (UNESCO, 1990, art. 5). Quince años más tarde, en la Declaración de Incheon (11) se reconoce que "es preciso aprovechar las tecnologías de la información y la comunicación (TIC) para reforzar los sistemas educativos, la difusión de conocimientos, el acceso a la información, el aprendizaje efectivo y de calidad, y una prestación más eficaz de servicios" (UNESCO, 2015, p. 3). De este modo, encontramos que las TIC empiezan a ser un recurso didáctico imprescindible dentro de los sistemas educativos.

Desde el marco DUA, como un enfoque práctico e inclusivo, se tiene como pretensión un diseño curricular flexible bajo entornos y experiencias de aprendizaje que contemplen la diversidad de los estudiantes. Para ello, el DUA se apoya en la versatilidad y flexibilidad de las TIC. Con la llegada del nuevo milenio, el Foro Mundial de Educación celebrado en Dakar (6) expone, entre varios compromisos, uno relacionado con las TIC: "aprovechar las nuevas tecnologías de la información y la comunicación para contribuir al logro de los objetivos de la Educación para Todos" (UNESCO, 2000, p. 9).

Seis años más tarde, la Convención sobre los derechos de las personas con discapacidad (7) reconoce en su artículo 9 "promover el acceso de las personas con discapacidad a los nuevos sistemas y tecnologías de la información y las comunicaciones, incluida Internet” (Naciones Unidas, 2006, art. 9, p. 11).

$\mathrm{Al}$ año siguiente, en la Declaración de Lisboa (8) se expone que "las TIC (Tecnologías de la Información y Comunicación) están mejorando y los libros digitales son de mayor calidad" (Agencia Europea para el Desarrollo de las Necesidades Educativas Especiales, 2007, p. 2). Posteriormente, la Agenda 2030 (10) reconoce en favor de las TIC lo siguiente: "la expansión de las tecnologías de la información y las comunicaciones y la interconexión mundial brinda grandes posibilidades para acelerar el progreso humano" (Naciones Unidas, 2015, p. 6).

Los resultados obtenidos en esta categoría desprenden una importante presencia en los documentos objeto de estudio de las TIC como un recurso y apoyo en la labor educativa.

A modo de síntesis, entre los documentos analizados, es el Foro Mundial (6) el que más se aproxima al paradigma DUA, aunque no contemple la totalidad de las categorías de análisis, resaltando "la elaboración de enfoques de aprendizaje múltiples, flexibles e innovadores y un entorno que fomente la confianza y el respeto mutuos" (UNESCO, 2000, p. 20). 
Bajo esta orientación el DUA se concibe como un enfoque que atiende a la flexibilidad en el diseño curricular y proporciona múltiples formas de representación, de acción y expresión y de motivación. Estos tres principios son la piedra angular del modelo DUA en su aplicación práctica en el aula.

\section{Conclusiones}

El análisis de los fundamentos relacionados con el DUA tanto a nivel de presencia como ausencia en los diferentes documentos revisados representa una cartografía del estado del DUA en el ámbito internacional del cual se deducen una serie de conclusiones.

En primer lugar, podemos concluir que los fundamentos del marco DUA no están presentes en su totalidad en los documentos publicados por los organismos internacionales. Es por ello, que podemos afirmar que el DUA debería tener un mayor reconocimiento y prestigio internacional, y que todavía tiene un largo recorrido para poder asentarse como un modelo de inclusión que cuente con el aval internacional y forme parte de los textos legales a nivel mundial. En esta línea, se propone que la implementación del DUA como herramienta para garantizar la inclusión, debe formar parte de las políticas educativas, lo que requiere de inversión en recursos humanos y materiales. Asimismo, debe incluirse en los planes de estudio de los futuros docentes que les capacite y entrene en materia de inclusión. Dentro del contexto escolar, el DUA debe ser seña de identidad del Proyecto Educativo de la institución. Sólo así, podrá existir un compromiso real y objetivo con la inclusión educativa. Bajo esta conclusión se desprende la idea de que la inclusión es una aspiración más que una realidad de los sistemas educativos. Aun así, cabe señalar que los organismos internacionales en materia de educación se inscriben en el marco de la política social a nivel mundial bajo los principios de inclusión y calidad como piedras angulares de una educación transformadora, y que los documentos que han publicado a lo largo de los años han ejercido influencia en las políticas de los países desarrollados y subdesarrollados, por ejemplo, poniendo en marcha el movimiento de una Educación para Todos. No obstante, actualmente seguimos encontrando países con carencias en términos de equidad, calidad, acceso a la educación e igualdad de oportunidades.

En segundo lugar, la Declaración Universal de los Derechos Humanos (1) (ONU, 1948), la Declaración de Educación Para Todos (3) (UNESCO, 1990) y el Marco de Acción sobre Necesidades Educativas Especiales. Salamanca (5) (UNESCO, 1994) han sido referencia base de los documentos revisados. Es notorio como los textos posteriores a ellos hacen alusiones de manera continuada a lo expuesto en estos tres documentos, siendo el documento que se desprende del Foro Mundial de Educación (6) (UNESCO, 2000), el que más se apoya a los fundamentos del DUA. No obstante, es significativo el nulo tratamiento que se ha dado por parte de las organizaciones internacionales a la neurociencia y su vinculación con la educación.

En tercer lugar, se deduce que a medida que se producen cambios en la sociedad, los organismos internacionales van acogiendo nuevos retos para garantizar una educación inclusiva y eliminar la exclusión social. Dos ejemplos de ello son: la inclusión de las TIC y la consideración de la igualdad de género.

Aunque este estudio se basa en los siete fundamentos principales del DUA, cabe señalar que estos se podrían extender a otras categorías, como por ejemplo la participación de 
la comunidad educativa. La delimitación se debe a la necesaria concreción para garantizar la viabilidad del estudio. Otro aspecto a señalar como limitación se refiere al contexto de análisis, siendo en este caso el ámbito internacional, dejando de lado lo referente a documentos de carácter normativo en materia educativa. En esta línea, se plantearían como posibles líneas de investigación la aplicación del estudio en la normativa educativa en España concretada en su Leyes Orgánicas y Reales Decretos, así como el análisis de la presencia de los fundamentos del DUA en el desarrollo curricular a nivel de Comunidad Autónoma. Estas líneas futuras de trabajo contribuirían a plasmar una visión completa del DUA en el sistema educativo para localizar tanto referencias como ausencias de dicho enfoque a nivel curricular y normativo.

\section{REFERENCIAS}

Agencia Europea para el desarrollo de la Educación Especial (2007). Declaración de Lisboa. Las opiniones de los jóvenes sobre Inclusión Educativa. Recuperado el 10 de marzo de 2020 de https://www. european-agency.org/sites/default/files/lisbon-declaration-young-people2019s-views-on-inclusiveeducation_declaration_es.pdf

Alba, C. (2019). Diseño Universal para el Aprendizaje: un modelo teórico práctico para una educación inclusiva de calidad. Revista Participación Educativa, 6(9), 55-66.

Blanco, R. (2006). La equidad y la inclusión social uno de los desafíos de la educación y la escuela de hoy. Revista Electrónica Iberoamericana sobre Calidad, Eficacia y Cambio en Educación, 4(3), 1-15.

Burgstahler, S. (2008). Universal design in higher education. Universal design in higher education: From principles to practice. Harvard Education Press.

Center for Universal Design (1997). The principles of Universal Design, version 2.0. North Carolina State University.

Centro de Tecnología Especial Aplicada (1984). Centro Especial de Tecnología Especial Aplicada. Retrieved from http://www.cast.org/udl/index.html

Centro de Tecnología Especial Aplicada (2011). Universal Design for Learning guidelines version 2.0. Recuperado el 10 de marzo de 2020 de http://www.cast.org/udl/index.html

Fernández-Batanero, J. M., \& Arrifano, T. P. (2019). Educación inclusiva y TIC. Revista Aula abierta, $48(2), 125-128$.

Hartmann, E. (2011). Diseño Universal para el Aprendizaje (UDL). Consorcio Nacional sobre sordoceguera. Perspectiva de Práctica. Destacando la información sobre sordoceguera, 8. Recuperado el 10 de marzo de 2020 de http://www.perkinsla.org/recursosbiblio/_1UDL-spanish.pdf

Instituto Universitario de Integración en la Comunidad (2009). Mejor Educación para todos, un informe mundial. Universidad de Salamanca.

Mace, R. (1985). Universal Design. Barrier Free Environments for Everyone. Designers West.

Mariussi, M. I., Gisi, M. L., \& Eyng, A. M. (2016). A Escola como Espaço para Efetivação dos Direitos Humanos das Pessoas com Deficiência. Revista Brasileira de Educação Especial, 22(3), 443-454. https://doi.org/10.1590/S1413-65382216000300010

Naciones Unidas para la Educación, la Ciencia y la Cultura (1990). Declaración Mundial sobre Educación para Todos: Satisfacción de las Necesidades Básicas de Aprendizaje. Recuperado el 10 de marzo de 2020 de https://unesdoc.unesco.org/ark:/48223/pf0000127583_spa 
Naciones Unidas para la Educación, la Ciencia y la Cultura (1994). Declaración de Salamanca y Marco de Acción sobre Necesidades Educativas Especiales. UNESCO/Ministerio de Educación de España. Recuperado el 10 de marzo de 2020 de https://unesdoc.unesco.org/ark:/48223/pf0000098427_spa

Naciones Unidas para la Educación, la Ciencia y la Cultura (2000). Marco de Acción de Dakar: Educación para Todos: cumplir nuestros compromisos comunes (con los seis marcos de acción regionales). Educación para Todos: cumplir nuestros compromisos comunes. Recuperado el 10 de marzo de 2020 de https:// unesdoc.unesco.org/ark:/48223/pf0000121147_spa

Naciones Unidas para la Educación, la Ciencia y la Cultura (2008). Conferencia Internacional de Educación "La Educación Inclusiva: el Camino hacia el futuro". Cuadragésima octava reunión. Ginebra, 25 a 28 de noviembre de 2008. Recuperado el 10 de marzo de 2020 de http://www.ibe.unesco.org/fileadmin/ user_upload/Policy_Dialogue/48th_ICE/General_Presentation-48CIE-4_Spanish_.pdf

Naciones Unidas para la Educación, la Ciencia y la Cultura (2015). Declaración de Incheon: Educación 2030: Hacia una Educación Inclusiva y Equitativa de Calidad y un Aprendizaje a lo Largo de la Vida para Todos Recuperado el 10 de marzo de 2020 de https://unesdoc.unesco.org/ark:/48223/ pf0000233137_spa?posInSet=1\&queryId=47a850f6-5687-4f56-9db5-062b7b97f15bb

Organización de las Naciones Unidas (1948). Declaración Universal de los Derechos humanos. Adoptada y proclamada por la Asamblea General en su resolución 217 A (III), de 10 de diciembre de 1948. Recuperado el 10 de marzo de 2020 de https://www.un.org/es/universal-declaration-human-rights/

Organización de las Naciones Unidas (1989). Convención de los Derechos del Niño de 20 de noviembre de 1989. Recuperado el 10 de marzo de 2020 de https:/www.un.org/es/events/childrenday/pdf/ derechos.pdf

Organización de las Naciones Unidas (1993). Normas uniformes sobre igualdad de oportunidades con discapacidad. Resolución Aprobada por la Asamblea General, Cuadragésimo octavo periodo de sesiones, de 20 de diciembre de 1993. Recuperado el 10 de marzo de 2020 de https://www.un.org/esa/socdev/ enable/dissres $0 . \mathrm{htm}$

Organización de las Naciones Unidas (2006). Convención sobre los derechos de las personas con discapacidad. Recuperado el 10 de marzo de 2020 de https://www.un.org/esa/socdev/enable/documents/ tccconvs.pdf

Organización de las Naciones Unidas (2015). Transformar nuestro mundo: la Agenda 2030 para el Desarrollo Sostenible. Resolución aprobada por la Asamblea General de las Naciones Unidas el 25 de septiembre de 2015 Recuperado el 10 de marzo de 2020 de https://unctad.org/meetings/es/ SessionalDocuments/ares70d1_es.pdf

Parra, D. C. (2011). Educación Inclusiva: un modelo de diversidad humana. Revista Educación y Desarrollo Social, 5(1), 139-150.

Payne, G., \& Payne, J. (2004). Key concepts in social research. London: Sage Publications.

Rose, D. H., \& Meyer, A. (2002). Teaching Every Student in the Digital Age. Alexandria: Association for Supervision \& Curriculum Debe - ASCD.

Rose, D. H., \& Meyer, A. (2006). A Practical Reader in Universal Design for Learning. Massachussets: Harvard Education Press.

Ruiz-Bel, R., Salas, L. S., Echeita, G., Sala-Bars, I., \& Datsira, M. (2012). El principio del Universal Design. Concepto y desarrollos en la enseñanza superior. Revista de Educación, 359, 413-430. 
Salas, R. (2003). La educación, ¿necesita realmente de la neurociencia?. Estudios Pedagógicos, 29, 155171. 10.4067 / S0718-07052003000100011

Schelly, C. L., Davies, P. L., \& Spooner, C. L. (2011). Student Perceptions of Faculty Implementation of Universal Design for Learning. Journal of Postsecondary Education and Disability, 24(1), 17-28.

Suárez, B., \& López, A. (2018). Investigación en Educación Inclusiva: la producción de trabajos en Revistas Españolas y Tesis Doctorales. Revista Nacional e Internacional de Educación Inclusiva, 11(1), 169.

Torres, G. J. (2010). Pasado, presente y futuro de la atención a las necesidades educativas especiales: Hacia una educación inclusiva. Perspectiva Educacional, 49(1), 62-89.

Universidad Nacional de Educación a Distancia (2018). La inclusión educativa como proceso en contextos socioeducativos. Madrid: UNED.

Watkins, A. (2009). Principios fundamentales para la promoción de la calidad de la educación inclusiva. Recomendaciones a responsables políticos. Agencia Europea para el Desarrollo de la Educación del Alumnado con Necesidades Educativas Especiales. Recuperado el 10 de marzo de 2020 de https:// www.european-agency.org/sites/default/files/key-principles-for-promoting-quality-in-inclusiveeducation_key-principles-ES.pdf

Zulian, M. S., \& Freitas, S. N. (2001). Formação de Professores na Educação Inclusiva: aprendendo a viver, criar, pensar e ensinar de outro modo. Revista Educação Especial, Santa Maria, 18, 1-7. http:// dx.doi.org/10.5902/1984686X

\section{REFERÊNCIAS DOS DOCUMENTOS UTILIZADOS EN LA REVISIÓN SISTEMÁTICA}

agencia Europea para el desarrollo de la Educación Especial (2007). Declaración de Lisboa. Las opiniones de los jóvenes sobre Inclusión Educativa. Recuperado el 10 de marzo de 2020 de https://www. european-agency.org/sites/default/files/lisbon-declaration-young-people2019s-views-on-inclusiveeducation_declaration_es.pdf

Naciones Unidas para la Educación, la Ciencia y la Cultura (1990). Declaración Mundial sobre Educación para Todos: Satisfacción de las Necesidades Básicas de Aprendizaje. Recuperado el 10 de marzo de 2020 de https://unesdoc.unesco.org/ark:/48223/pf0000127583_spa

Naciones Unidas para la Educación, la Ciencia y la Cultura (1994). Declaración de Salamanca y Marco de Acción sobre Necesidades Educativas Especiales. UNESCO/Ministerio de Educación de España. Recuperado el 10 de marzo de 2020 de https://unesdoc.unesco.org/ark:/48223/pf0000098427_spa

Naciones Unidas para la Educación, la Ciencia y la Cultura (2000). Marco de Acción de Dakar: Educación para Todos: cumplir nuestros compromisos comunes (con los seis marcos de acción regionales). Educación para Todos: cumplir nuestros compromisos comunes. Recuperado el 10 de marzo de 2020 de https:// unesdoc.unesco.org/ark:/48223/pf0000121147_spa

Naciones Unidas para la Educación, la Ciencia y la Cultura (2008). Conferencia Internacional de Educación "La Educación Inclusiva: el Camino hacia el futuro". Cuadragésima octava reunión. Ginebra, 25 a 28 de noviembre de 2008. Recuperado el 10 de marzo de 2020 de http://www.ibe.unesco.org/fileadmin/ user_upload/Policy_Dialogue/48th_ICE/General_Presentation-48CIE-4_Spanish_.pdf

Naciones Unidas para la Educación, la Ciencia y la Cultura (2015). Declaración de Incheon: Educación 2030: Hacia una Educación Inclusiva y Equitativa de Calidad y un Aprendizaje a lo Largo de la 
Vida para Todos Recuperado el 10 de marzo de 2020 de https://unesdoc.unesco.org/ark:/48223/ pf0000233137_spa?posInSet=1 \&queryId=47a850f6-5687-4f56-9db5-062b7b97f15bb

Organización de las Naciones Unidas (1948). Declaración Universal de los Derechos humanos. Adoptada y proclamada por la Asamblea General en su resolución 217 A (III), de 10 de diciembre de 1948. Recuperado el 10 de marzo de 2020 de https://www.un.org/es/universal-declaration-human-rights/

Organización de las Naciones Unidas (1989). Convención de los Derechos del Niño de 20 de noviembre de 1989. Recuperado el 10 de marzo de 2020 de https:/www.un.org/es/events/childrenday/pdf/ derechos.pdf

Organización de las Naciones Unidas (1993). Normas uniformes sobre igualdad de oportunidades con discapacidad. Resolución Aprobada por la Asamblea General, Cuadragésimo octavo periodo de sesiones, de 20 de diciembre de 1993. Recuperado el 10 de marzo de 2020 de https://www.un.org/esa/socdev/ enable/dissres $0 . \mathrm{htm}$

Organización de las Naciones Unidas (2006). Convención sobre los derechos de las personas con discapacidad. Recuperado el 10 de marzo de 2020 de https://www.un.org/esa/socdev/enable/documents/ tccconvs.pdf

Organización de las Naciones Unidas (2015). Transformar nuestro mundo: la Agenda 2030 para el Desarrollo Sostenible. Resolución aprobada por la Asamblea General de las Naciones Unidas el 25 de septiembre de 2015 Recuperado el 10 de marzo de 2020 de https://unctad.org/meetings/es/ SessionalDocuments/ares70d1_es.pdf

\section{Errata}

Nas páginas de 269 a 284

Onde se lê: AVELINO, M.O.A. \& FERRAZ, P.C.S.

Leia-se: CORTÉS DÍAZ, M., FERREIRA VILLA, C., \& ARIAS GAGO, A. R.”

Recebido em: 10/04/2020

Reformulado em: 10/07/2020

Aprovado em: 24/07/2020 